\title{
Inter-Relações da Violência no Sistema Familiar: Estudo Domiciliar em um Bairro de Baixa Renda
}

\author{
Interrelationships of Violence in the Family System: a Household Survey \\ in a Low-Income Neighborhood
}

\author{
Fernanda Monteiro de Castro Bhona* ${ }^{*}$, Carla Ferreira de Paula Gebara ${ }^{b}$, Ana Regina Noto ${ }^{b}$ \\ Marcel de Toledo Vieira ${ }^{c} \&$ Lelio Moura Lourenço ${ }^{c}$ \\ ${ }^{a}$ Tribunal de Justiça de Minas Gerais, Juiz de Fora, Minas Gerais, Brasil, \\ ${ }^{b}$ Universidade Federal de São Paulo, São Paulo, São Paulo, Brasil \\ \& ' Universidade Federal de Juiz de Fora, Juiz de Fora, Minas Gerais, Brasil
}

\begin{abstract}
Resumo
Visando identificar associações entre tipos de violência na família, realizou-se um levantamento domiciliar por amostragem probabilística com 480 mulheres, entre 18 e 60 anos $(M=39,1 ; D P=12,2)$, num bairro de baixa renda em Juiz de Fora/MG. Utilizou-se a Escala de Táticas de Conflito Revisada e a Escala de Táticas de Conflito entre Pais-Crianças. A violência psicológica variou de $77 \%$ a $89 \%$ nas relações estudadas. A violência física foi maior ou igual a $20 \%$. Foram observadas associações entre praticar e ser vítima de quase todas as modalidades de violência conjugal. Ser vítima ou agressora na relação conjugal também apresentou associação com violência contra filhos. Tais resultados evidenciam a circularidade das relações violentas e a relevância de ações preventivas na família em detrimento a ações individualizadas.

Palavras-chave: Violência na família, conflito conjugal, abuso infantil, relações familiares.
\end{abstract}

\begin{abstract}
Aiming the identification of associations between different types of family violence, we performed a household survey using a probabilistic sample of 480 women, aged between 18 and 60 years $(M=39.1 ; S D=12.2)$, from a low-income neighborhood of Juiz de Fora/MG. The Revised Conflict Tactics Scales and the Parent-Child Conflict Tactics Scales were applied. Psychological violence was reported in $77 \%$ to $89 \%$ of the relationships investigated. Physical violence was higher or equal to $20 \%$. Associations between practicing and being victim of almost all types of partner violence were observed. Being victim or aggressor in the marital relationship was also associated with violence against children. These results highlight the circularity of violence and the relevance of preventive actions in the family.
\end{abstract}

Keywords: Family violence, marital conflict, child abuse, family relations.

Violência, segundo a Organização Mundial de Saúde (OMS), pode ser definida como:

o intencional uso da força física ou do poder, em ameaça ou real, contra si próprio, outra pessoa, contra um grupo ou comunidade, que resulte ou tenha probabilidade de resultar em injúria, morte, dano psicológico, privação ou prejuízos no desenvolvimento. (Krug, Dahlberg, Mercy, Zwi, \& Lozano, 2002, p. 5)

* Endereço para correspondência: Tribunal de justiça de Minas Gerais, Av. Rio Branco, 2189, 12 andar, Centro, Juiz de Fora, MG, Brasil 36025-000. E-mail: fbhona@ gmail.com

Agradecimentos às agências de fomento Fundação de Amparo à Pesquisa do Estado de São Paulo (FAPESP; processos $n^{\circ}: 2010 / 51094-7$ e $n^{\circ}: 10 / 518370$ ) e Conselho Nacional de Desenvolvimento Científico e Tecnológico (CNPq; processo ${ }^{\circ} 400675 / 2010-2$ ) pelos recursos que viabilizaram a realização da pesquisa apresentada.
O comportamento violento relaciona-se a sérias implicações para a saúde, bem como para o desenvolvimento psicológico e social de indivíduos, famílias e comunidades (Krug et al., 2002).

Quando tais condutas acontecem entre pessoas que possuem vínculos familiares, utiliza-se a denominação violência doméstica ou intrafamiliar. Essa expressão, por sua amplitude, engloba a violência que acontece entre parceiros íntimos, ou conjugal; a violência direcionada aos filhos; a violência contra idosos; e outras possíveis no contexto das relações de afeto (Minayo, 2005). A forma através da qual o comportamento violento se materializa nas relações interpessoais pode ser: física (com uso da força física na intenção de causar dano a outrem); psicológica (por meio de agressões verbais, gestuais, ameaças, por exemplo); sexual (envolve a imposição de práticas de cunho sexual contra a vontade da pessoa ou que propiciem 
sua vitimização); e também através da privação ou negligência (ausência ou recusa de cuidados a quem necessita; Krug et al., 2002; Minayo, 2005).

No que se refere à violência entre parceiros íntimos, observa-se o maior destaque dado à mulher vitimizada na relação de casal (Krug et al., 2002). Porém, há o reconhecimento de que a mulher também pode ser violenta numa relação conjugal (Archer, 2002; Vieira et al., 2008), sendo desejável a investigação da ocorrência de violência mútua, preferencialmente a partir dos relatos de ambos os envolvidos (Testa, Livingston, \& Leonard, 2003; Zaleski, Pinsky, Laranjeira, Ramisetty-Mikler, \& Caetano, 2010).

Quanto à vitimização de crianças e adolescentes no contexto da família, os agressores do sexo feminino são os mais freqüentes, e os do sexo masculino costumam ser autores das agressões mais violentas, provocando lesões e sequelas mais graves (Canha, 2008). Estudos sobre o assunto indicam que agressões são consideradas práticas disciplinares necessárias adotadas nas famílias, reproduzidas através de gerações (Martins \& Jorge, 2009; Vendruscolo, Ferriani, \& Silva, 2007; Zanoti-Jeronymo et al., 2009).

O estudo teórico da agressividade humana, na perspectiva da aprendizagem social, considera que o comportamento violento pode ser aprendido nas interações sociais através, até mesmo, da simples observação de modelos (Bandura, Ross, \& Ross, 1961). Além disso, contemplando uma análise do ambiente no qual a violência emerge, bem como da multicausalidade que caracteriza o fenômeno, a perspectiva bioecológica do desenvolvimento humano tem sido útil na compreensão das condutas violentas (Carvalho-Barreto, Bucher-Maluschke, Almeida, \& DeSouza, 2009; Krug et al., 2002).

Proposto por Urie Bronfenbrenner, o modelo bioecológico descreve a importância das interações recíprocas entre pessoas (e destas com objetos e símbolos), e dos ambientes como contextos de desenvolvimento, sendo estes importantes elementos na formação de características e competências humanas. As interações entre indivíduos, progressivamente mais complexas, com o envolvimento ativo das pessoas envolvidas e, ocorrendo durante um tempo prolongado, constituem os chamados processos proximais, considerados os motores do desenvolvimento. A influência desses processos no desenvolvimento varia em função de características da pessoa, do ambiente em que ocorre (imediato e mais remoto), e da característica em questão. (Bronfenbrenner, 1994; Bronfenbrenner \& Evans, 2000). Assim, em síntese é possível afirmar que são analisados diferentes níveis de características e influências que atuam na formação dos indivíduos, os elementos: pessoa, processo, contexto e tempo (PPCT; Bronfenbrenner \& Evans, 2000; Carvalho-Barreto et al., 2009).

Cabe destacar que o ambiente nesse modelo é considerado de forma ampla, em diversas dimensões que se interrelacionam e se sobrepõem: microssistema, mesossistema, exossistema e macrossistema. O microssistema envolve a pessoa e suas relações, incluindo as interações face-a-face, em determinado local (setting), considerando- -se suas características físicas, as atividades desenvolvidas e os papéis desempenhados, em um dado período de tempo. O mesossistema compreende as relações entre os diferentes locais que a pessoa frequenta em determinado momento de sua vida (por exemplo, durante a infância: casa, escola, igreja). O exossistema, como uma extensão do mesossistema, abrange outras estruturas sociais, nas quais a pessoa não se envolve diretamente, mas recebe suas influências nos contextos em que se insere. Diferente das dimensões anteriores, o macrossistema não necessariamente se materializa em ambientes concretos. É composto por um abrangente padrão de características de uma dada cultura, sistema de crenças, estilo de vida (Bronfenbrenner, 1977, 1994).

Quando considera-se as condutas de violência na família, é importante destacar que tais eventos impactam não somente a vítima direta da agressão, mas os demais membros que constituem esse microssistema, no qual operam os processos proximais, promotores do desenvolvimento (Holt, Buckley, \& Whelan, 2008; Reichencheim, Dias, \& Moraes, 2006). A existência ou não de apoio familiar e social às vítimas que pode advir do mesossistema (vizinho, parentes, escola), bem como do exossistema (instituições públicas, conselho tutelar, serviços de saúde) são aspectos que podem ou não favorecer a perpetuação dessas condutas na família (De Antoni \& Koller, 2011). A aceitabilidade social da violência como estratégia disciplinar na educação dos filhos ou como recurso válido nos conflitos conjugais é um dos fatores do macrossistema que interfere em tais situações (Martins \& Jorge, 2009).

No que diz respeito à dimensão tempo, o modelo indica a necessidade de serem consideradas também as mudanças e continuidades nas pessoas e nos ambientes que frequentam (Bronfenbrenner, 1994; Bronfenbrenner \& Evans, 2000). O histórico de violência na família de origem e sua reprodução em gerações posteriores também é aspecto analisado em alguns estudos sobre o tema (Carvalho-Barreto et al., 2009; Cecconello, De Antoni, \& Koller, 2003).

Contudo, Stelko-Pereira e Wiliams (2010) apontam que os estudos na área de violência doméstica tendem a focalizar determinados grupos considerados mais vulneráveis (mulheres, crianças e idosos, por exemplo) ou se centrar em díades específicas. A partir do entendimento de que a violência que acontece no contexto das relações familiares é fenômeno complexo, que apresenta consequências e reflexos no desenvolvimento que vão além de díades e relações unidirecionais (Salisbury, Henning, \& Holdford, 2009), buscou-se: (a) investigar a prevalência desses eventos em mulheres residentes em um bairro de baixa renda, tanto no que se refere aos seus parceiros, quanto nas relações com seus filhos; (b) identificar associações entre diferentes tipos de violência no sistema familiar.

Estudos com informações sobre como esses fenômenos se apresentam em determinada população, em amostras comunitárias, ainda são escassos e recentes no país (Oliveira et al., 2009; Zaleski et al., 2010; Zanoti-Jeronymo 
Bhona, F. M. C., Gebara, C. F. P., Noto, A. R., Vieira, M. T. \& Lourenço, L. M. (2014). Inter-Relações da Violência no Sistema Familiar: Estudo Domiciliar em um Bairro de Baixa Renda.

et al., 2009). A delimitação de um perfil socioeconômico específico foi adotada uma vez que algumas pesquisas apontam essa característica como possível fator associado a situações de violência (Abramsky et al., 2011; Oliveira et al., 2009; Zanoti-Jeronymo et al., 2009). Os dados do presente estudo podem contribuir na elaboração de estratégias de intervenção e prevenção da violência junto a famílias, favorecendo a promoção de experiências mais positivas no desenvolvimento de indivíduos nesse importante contexto de socialização.

\section{Método}

\section{Contexto da Pesquisa}

Dados do Centro de Referência em Assistência Social que atende o bairro pesquisado indicam que dos 11.667 moradores adultos, cerca de 4.414 pessoas foram cadastradas para receber algum tipo de assistência governamental. O local é atendido por serviços de transporte público, creche comunitária, rede de ensino estadual e municipal, unidade de saúde, posto policial e comércio regional.

\section{Delineamento}

A pesquisa aqui descrita é parte de um projeto mais amplo que investiga relações entre o consumo de álcool, violência doméstica e aspectos relativos à saúde (física $\mathrm{e}$ mental) da mulher. Neste artigo são apresentadas somente as investigações pertinentes à violência na família. Para tanto, foi realizado um levantamento de domicílios por amostragem probabilística a fim de assegurar a representatividade dos resultados e a realização de inferência estatística válida para a população alvo do estudo (mulheres residentes em um bairro de baixa renda da cidade de Juiz de Fora-MG).

Para determinação do bairro de baixa renda a ser pesquisado, foi aleatoriamente selecionado um bairro em Juiz de Fora cujo rendimento médio mensal por pessoa estivesse situado entre o $20^{\circ}$ e o $30^{\circ}$ percentis, segundo os dados do Censo Demográfico do Instituto Brasileiro de Geografia e Estatística (IBGE), realizado em 2000. Essa foi a forma escolhida para retratar o perfil socioeconômico desejado sem abordar situações extremas. No momento da elaboração do desenho amostral do estudo, os dados do Censo Demográfico do IBGE de 2012 ainda não estavam disponíveis.

\section{Participantes}

As mulheres domiciliadas na região do bairro sorteado, na faixa etária de 18 a 60 anos de idade, alfabetizadas, que não apresentaram comprometimento cognitivo evidente, formaram a população alvo do estudo. Foram entrevistadas ao todo 480 mulheres, dentre as quais 252 possuíam filhos com idade até 18 anos, e 292 encontravam-se em um relacionamento conjugal, seja com marido ou com companheiro, residente no mesmo domicílio.

O cálculo da amostra considerou 3\% de erro máximo desejado para a pesquisa, nível de confiança de $95 \%$, correção para populações finitas, efeito do plano amostral igual a 2, e possíveis perdas por recusa como aproximadamente $25 \%$. Foi adotado o método de amostragem por conglomerados em dois estágios. As ruas pertencentes ao bairro foram consideradas como unidades primárias de amostragem, e os domicílios, como unidades secundárias de amostragem. Para a seleção das ruas, foi adotado o método de amostragem aleatória simples sem reposição, enquanto os domicílios foram selecionados por amostragem sistemática.

$\mathrm{Na}$ amostra pesquisada $281(58,5 \%)$ mulheres eram brancas, $298(62,1 \%)$ eram católicas, e $292(60,8 \%)$ exerciam atividade profissional. A média de idade foi de 39,1 anos. Quanto à escolaridade, $239(49,8 \%)$ mulheres apresentaram instrução até o ensino fundamental, 186 $(38,8 \%)$ tinham cursado o ensino médio e 55 (11\%) possuíam instrução de nível superior.

\section{Instrumentos}

As participantes responderam às questões contidas em três instrumentos: questionário sociodemográfico, e duas escalas de violência doméstica. A primeira escala de violência foi a Escala de Táticas de Conflito Revisada (adaptação da Revised Conflict Tactics Scales - CTS2), que identifica condutas agressivas nas relações de casal. A Escala de Táticas de Conflito entre Pais e Crianças (adaptação da Parent-Child Conflict Tactics Scales - CTSPC) foi utilizada para identificar comportamentos de violência das mulheres em relação aos filhos.

O questionário sociodemográfico elaborado especialmente para a pesquisa, possui 21 itens destinados a descrever a amostra a partir de características consideradas relevantes em estudos anteriores sobre o assunto, tais como: idade, raça, religião, escolaridade, situação profissional e conjugal, caracterização socioeconômica (Abramsky et al., 2011; Lamoglia \& Minayo, 2009). As duas escalas de violência, elaboradas por Straus e colaboradores nos anos 90, foram avaliadas previamente em amostras brasileiras no que tange à equivalência conceitual, semântica e propriedades psicométricas (Moraes, Hasselmann, \& Reichencheim, 2002; Moraes \& Reichencheim, 2002; Reichencheim, Klein, \& Moraes, 2007; Reichencheim \& Moraes, 2003, 2006). Em ambas a maioria das estimativas de confiabilidade intraobservador (kappa) apresentou pontuação acima de 0,75 , e a consistência interna variou de 0,49-0,68 na CTSPC, e de 0,65-0,86 na CTS2 (Moraes \& Reichencheim, 2002; Reichencheim \& Moraes, 2006).

A Escala de Táticas de Conflito Revisada (CTS2), com 78 itens, descreve possíveis comportamentos do respondente e de seu companheiro. É composta por cinco subescalas: negociação, agressão psicológica, violência física, consequências da violência sobre a saúde do respondente e de seu companheiro (injúria) e coerção sexual entre o casal. Neste estudo não foram analisadas as respostas pertinentes a condutas de negociação por não abordarem comportamentos violentos de fato. 
A Escala de Táticas de Conflito entre Pais e Crianças (CTSPC), através de 22 questões, aborda condutas de pais em relação a filhos, as quais são agrupadas em três subescalas: disciplina não-violenta, agressão psicológica e violência física. Esta última é apresentada no instrumento através de itens relacionados à punição corporal (que aborda atos menos severos de violência física); maus-tratos físicos e maus-tratos físicos graves. Diante da semelhança com relação à gravidade entre as condutas de maus-tratos físicos e maus-tratos físicos graves, tais comportamentos foram agrupados em uma única categoria para fins de análise (maus-tratos físicos). Como o foco do trabalho foi observar o comportamento violento, os itens de disciplina não violenta não foram analisados inicialmente.

Em ambas as escalas de violência a respondente tem oito opções de resposta, as quais indicam o número de vezes que determinado comportamento ocorreu $(1=$ uma vez nos últimos três meses; $2=$ duas vezes nos últimos três meses; 3 = três a cinco vezes nos últimos três meses; $4=$ seis a dez vezes nos últimos três meses; $5=$ onze a vinte vezes nos últimos três meses; $6=$ mais que vinte vezes nos últimos três meses; $7=$ não aconteceu nos últimos três meses mas já aconteceu antes; 8 = isso nunca aconteceu). Salienta-se que o lapso temporal especificado (três meses) atendeu às necessidades do projeto mais amplo no qual este estudo está inserido, e é passível de adaptações, conforme previsto no instrumento original que tem como referência o lapso temporal de um ano (Straus, 2001; Straus, Hamby, Boney-McCoy, \& Sugarman, 1996).

As respostas obtidas nas escalas de violência foram codificadas e analisadas sob a forma de variáveis categóricas. Os dados de prevalência foram considerados caso a respondente admitisse a ocorrência de pelo menos um dos tipos de violência investigados, ao menos uma vez na vida, forma de categorização prevista nas escalas originais (Straus, 2001; Straus et al., 1996). A frequência e severidade das ações não foram abordadas neste trabalho.

\section{Procedimentos}

Após aprovação pelo comitê de ética, a coleta de dados teve início no mês de maio de 2011 e foi concluída em outubro do mesmo ano. Foi realizada por uma equipe de alunas da graduação do curso de psicologia, as quais receberam treinamento específico para a pesquisa.

Cada participante foi abordada em seu domicílio, onde o questionário sociodemográfico foi aplicado em forma de entrevista, e as escalas de violência foram preenchidas diretamente pelas participantes, garantindo-se maior privacidade nessas questões. Inicialmente receberam uma carta de apresentação contendo informações gerais sobre a pesquisa, e na sequência foram apresentados os Termos de Consentimento Livre e Esclarecido (TCLE). A partir da concordância, foram aplicados os instrumentos na seguinte ordem: questionário sociodemográfico, escala de violência entre parceiros, escala de violência contra filhos. O tempo médio para aplicação foi de cerca de 30 minutos. Ao final da sua participação, cada mulher recebeu um fo- lheto informativo sobre serviços de saúde e de assistência psicossocial para situações de violência doméstica em funcionamento na região.

As pesquisadoras aplicaram os instrumentos em local o mais isolado possível dentro do domicílio, cuidados que pesquisas sobre o tema têm utilizado buscando a preservação das possíveis vítimas. Não houve a utilização da palavra "violência" nos instrumentos e abordagens de pesquisa, sendo tais comportamentos apenas descritos, mas não qualificados como tal. Essa opção metodológica baseia-se na percepção de que algumas mulheres não nomeiam situações de violência doméstica como violência, provavelmente devido às representações associadas ao termo (Schraiber, D’Oliveira, França, \& Pinho, 2002).

Os dados foram processados e analisados através do software estatístico Statistical Package for the Social Sciences (SPSS). Realizaram-se análises estatísticas bivariadas com o objetivo de avaliar a associação entre os diferentes tipos e relações de violência doméstica. Testes de Qui-quadrado de Pearson foram ajustados para considerar as características do desenho amostral adotado (Skinner, Holt, \& Smith, 1989), implementado no módulo Complex Samples do software SPSS.

Tabela 1

Caracterização Sociodemográfica das Participantes $(n=480)$

\begin{tabular}{lcc}
\hline \multicolumn{1}{c}{ Variável } & $f$ & $\%$ \\
\hline Faixa etária & & \\
18 a 29 anos & 123 & 25,6 \\
30 a 39 anos & 126 & 26,3 \\
40 a 49 anos & 115 & 23,9 \\
50 a 60 anos & 116 & 24,2 \\
Religião & & \\
Não tem & 24 & 5,0 \\
Católica & 298 & 62,1 \\
Evangélica/protestante & 132 & 27,5 \\
Outras & 25 & 5,2 \\
Escolaridade & & \\
Fundamental incompleto/completo & 239 & 49,8 \\
Ensino médio incompleto/completo & 186 & 38,8 \\
Superior completo incompleto/completo & 55 & 11,4 \\
Estado Civil & & \\
Casada/União estável & 292 & 60,8 \\
Outros (sem companheiro) & 188 & 39,2 \\
Filhos & & \\
Mulheres com filhos de até 18 anos & 252 & 52,5 \\
Mulheres sem filhos de até 18 anos & 228 & 47,5 \\
\hline
\end{tabular}


Bhona, F. M. C., Gebara, C. F. P., Noto, A. R., Vieira, M. T. \& Lourenço, L. M. (2014). Inter-Relações da Violência no Sistema Familiar: Estudo Domiciliar em um Bairro de Baixa Renda.

\section{Resultados}

Dentre os 781 domicílios abordados, 169 não apresentavam mulheres elegíveis para a pesquisa, ou não possuíam mulheres dentro da faixa etária do estudo, ou eram ocupados apenas por homens. Nos 512 domicílios restantes, obteve-se 480 entrevistas que fizeram parte do projeto mais amplo (consumo de álcool e violência). A taxa de resposta por domicílio foi de 72,6\%. A caracterização sociodemográfica das participantes é apresentada na Tabela 1.

Ressalta-se que nesse universo de 480 mulheres, 292 mulheres responderam a escala de violência conjugal, uma vez que declararam residir junto a um companheiro. As participantes com filhos até 18 anos, que responderam as questões pertinentes à violência contra filhos, correspondem a um total de 252 mulheres.

As prevalências de violência entre parceiros tendo a mulher como agressora indicaram: $77 \%$ de violência psi- cológica, $24,7 \%$ de violência física, $14,5 \%$ de violência sexual e $9,5 \%$ de injúria. Os relatos da violência praticada pelos homens contra as parceiras foram de: $71 \%$ de violência psicológica, 19,9\% de violência física, 16,4\% de violência sexual e $13,3 \%$ de injúria. Já a violência contra os filhos indicou 89,4\% de agressão psicológica, 77\% de punição corporal e 20,3\% de maus-tratos físicos.

Visando identificar as associações entre os diferentes episódios de violência na família testou-se, separadamente, a associação de cada tipo de violência praticada e sofrida pela mulher considerando-se sua relação com o companheiro e com os filhos. Destaca-se que para a realização dessas análises foram consideradas apenas as 190 mulheres que tinham companheiro e filhos com idade até 18 anos. Esses dados são apresentados na Tabela 2 e indicaram associação entre vivenciar violência na relação de casal (seja como vítima seja como agressora) e adotar condutas semelhantes com os filhos.

Tabela 2

Associações entre os Diferentes Tipos de Violência Doméstica Praticados e Sofridos pela Mulher, Considerando as Mulheres Com Companheiro e Com Filhos de Até 18 Anos de Idade (n=190)

\begin{tabular}{lcccccc}
\hline & \multicolumn{5}{c}{ Violência contra filhos } \\
\cline { 2 - 7 } & \multicolumn{2}{c}{ Psicológica } & Punição Corporal & Maus-Tratos Físicos \\
\hline & $x^{2}$ & $p$ & $x^{2}$ & $p$ & $x^{2}$ & $p$ \\
\hline Violência contra o homem & & & & & & \\
Psicológica & 17,474 & $<0,001$ & 11,051 & 0,004 & 12,654 & 0,002 \\
Física & 4,907 & 0,005 & 6,645 & 0,031 & 4,039 & 0,084 \\
Sexual & 2,256 & 0,055 & 2,818 & 0,142 & 1,756 & 0,251 \\
Injúria & 2,288 & 0,201 & 0,057 & 0,849 & 10,300 & 0,010 \\
Violência contra a mulher & & & & & & \\
Psicológica & 8,212 & 0,008 & 2,647 & 0,161 & 8,347 & 0,092 \\
Física & 1,678 & 0,110 & 4,359 & 0,059 & 2,044 & 0,194 \\
Sexual & 0,143 & 0,732 & 4,002 & 0,101 & 1,724 & 0,247 \\
Injúria & 1,418 & 0,138 & 0,022 & 0,901 & 1,951 & 0,217 \\
\hline
\end{tabular}

Nota $x^{2}=$ Valor ajustado com a correção de Rao-Scott com o objetivo de considerar as características do desenho amostral.

No que tange à violência entre parceiros, também foram testadas as associações entre vitimização e perpetração. Foram encontradas associações estatisticamente significativas $(p<0,01)$ entre agredir e ser vítima em quase todas as modalidades de violência investigadas (psicológica, física, sexual e injúria), conforme pode ser verificado na Tabela 3 .

\section{Discussão}

As informações obtidas indicam que condutas violentas na família apresentam prevalências consideráveis no universo pesquisado. Além de vítimas, as mulheres também se declararam agressoras dos companheiros e dos filhos, sendo constatadas associações significativas entre essas diferentes práticas no sistema familiar. 
Tabela 3

Associações entre os Tipos de Violência Praticadas entre Parceiros Íntimos Considerando as Mulheres Com Companheiro $(n=292)$

\begin{tabular}{|c|c|c|c|c|c|c|c|c|}
\hline & \multicolumn{8}{|c|}{ Violência contra a mulher } \\
\hline & \multicolumn{2}{|c|}{ Física } & \multicolumn{2}{|c|}{ Psicológica } & \multicolumn{2}{|c|}{ Sexual } & \multicolumn{2}{|c|}{ Injúria } \\
\hline & $x^{2}$ & $p$ & $x^{2}$ & $p$ & $x^{2}$ & $p$ & $x^{2}$ & $p$ \\
\hline \multicolumn{9}{|c|}{ Violência contra o homem } \\
\hline Física & 103,098 & $<0,001$ & 16,493 & $<0,001$ & 20,642 & $<0,001$ & 69,098 & $<0,001$ \\
\hline Psicológica & 31,654 & $<0,001$ & 153,592 & $<0,001$ & 10,836 & 0,001 & 11,769 & $<0,001$ \\
\hline Sexual & 25,368 & $<0,001$ & 4,295 & 0,076 & 80,816 & $<0,001$ & 10,687 & $<0,004$ \\
\hline Injúria & 84,659 & $<0,001$ & 12,551 & 0,003 & 18,530 & 0,001 & 164,061 & $<0,001$ \\
\hline
\end{tabular}

Nota $. \mathrm{x}^{2}=$ Valor ajustado com a correção de Rao-Scott com o objetivo de considerar as características do desenho amostral.

As prevalências de violência física e psicológica entre casais encontradas no presente estudo convergem com os resultados de outra investigação, de representatividade nacional, realizada no Brasil (Reichencheim, Moraes, Szklo, et al., 2006). No que se refere à prevalência de injúria, estudo com população representativa do município de São Paulo apresentou resultados no sentido do aqui constatado. Os pesquisadores encontraram percentual de $25,5 \%$ de mulheres com necessidade de procurar ajuda médica devido a brigas com o parceiro, e de 3,8\% de homens apresentando essa necessidade. Contudo destaca-se que esse estudo partiu de relatos de homens e mulheres (Oliveira et al., 2009), sendo importante destacar as diferenças existentes entre os gêneros no que tange à denúncia e consequências desse tipo de violência (Lamoglia \& Minayo, 2009; Thompson \& Kingree, 2006).

A prevalência de violência sexual contra mulheres não foi identificada em estudos de representatividade nacional. Entretanto, Schraiber et al. (2007) encontraram em uma região rural do Nordeste, índice próximo (14,3\%) ao obtido nesta investigação $(16,4 \%)$.

Sobre violência física contra filhos, as mulheres entrevistadas admitiram o uso de punições corporais em 77\% dos casos, e maus-tratos físicos em $20,3 \%$ da amostra. Levantamento de representatividade nacional no Brasil, que ouviu vítimas, encontrou prevalências de $33,8 \%$ para punição corporal e 10,3\% para maus-tratos físicos (Zanoti-Jeronymo et al., 2009). As maiores prevalências obtidas neste estudo sugerem que na localidade pesquisada tais práticas podem ser mais comuns que no país como um todo, o que indica a necessidade de programas de intervenção sobre o assunto na região.

Em relação à violência psicológica contra filhos, Moura e Reichencheim (2005), também encontraram altas prevalências desse tipo de violência (94\%) num ambulatório pediátrico. Os autores apontam que essas condutas de violência, menos evidentes, possuem potencial para se tornarem mais graves no futuro, sendo necessária a adoção de estratégias preventivas acerca do problema.
No que tange aos níveis de análise da abordagem bioecológica, no nível individual na amostra pesquisada, algumas das características sociodemográficas (tais como etnia, religião, estado civil, inserção no mercado de trabalho, renda, escolaridade), podem representar fatores de risco ou de proteção para eventos violentos, conforme a literatura sobre o assunto (Abramsky et al., 2011; D'Oliveira et al., 2009). Considerando-se que o fator renda pode estar relacionado aos fenômenos alvo da investigação (Carvalho-Barreto et al., 2009; Oliveira et al. 2009; Vieira et al., 2008), uma segunda etapa do estudo prevê comparativo futuro com dados coletados em um bairro de maior renda na cidade de Juiz de Fora -MG.

No que se refere às associações observadas, os dados obtidos indicam que determinado tipo de comportamento violento adotado por um membro da relação familiar pode ser direcionado a outras pessoas desse grupo, refletindo padrões relacionais de um indivíduo. Esse parece ser o caso das associações constatadas entre violência física e psicológica praticada pela mulher contra o parceiro e a utilização dessas mesmas práticas contra os filhos. Estudo de revisão de Swan, Gambone, Caldwell, Sullivan e Snow (2008) indica que a mulher, de forma tão frequente quanto o homem, tende a praticar violência física e psicológica contra o parceiro. Considerando-se a família, esses dados sugerem que essa conduta da mulher pode também ser reproduzida de forma frequente com a prole, o que é corroborado pelos registros nacionais oficiais, os quais indicam que as mães são as prováveis autoras das agressões contra crianças de até nove anos de idade em cerca de $60 \%$ de todos os casos de violência notificados (Assis, Avanci, Pesce, Pires, \& Gomes, 2012).

Além disso, as associações indicaram a possibilidade de uma mesma pessoa ser vítima e também agressora em diferentes relações, e que o tipo de violência sofrida se relaciona com o tipo de violência perpetrada. Na amostra obtida, ser vítima de violência psicológica e praticar violência física mais grave contra o parceiro (injúria) demonstrou relação com praticar o mesmo tipo de conduta 
Bhona, F. M. C., Gebara, C. F. P., Noto, A. R., Vieira, M. T. \& Lourenço, L. M. (2014). Inter-Relações da Violência no Sistema Familiar: Estudo Domiciliar em um Bairro de Baixa Renda.

com os filhos (violência psicológica e maus-tratos). Esses achados sugerem que a relação da mulher com o parceiro pode servir como modelo para sua relação com os filhos (Bandura, 2008).

Por fim, foram encontradas associações estatisticamente significativas entre praticar e ser vítima de quase todas as modalidades de violência entre parceiros investigadas. Tais resultados nesse tipo de díade sugerem a possibilidade de reciprocidade dos comportamentos violentos, como previsto na tipologia de violência entre parceiros íntimos proposta por Johnson (2009), a qual considera a possibilidade da chamada resistência violenta. Pesquisa conduzida por Smith, Homish, Leonard e Cornelius (2012) destaca a importância de se discutir a mutualidade na identificação das condutas de violência entre parceiros.

Essas relações de violência na família assinaladas (ho$\mathrm{mem} /$ mulher; mulher/homem; mulher/filhos) evidenciam os múltiplos impactos e direções desse tipo de conduta entre seus membros, o que corresponde ao nível processual de análise (grau das interações) na abordagem bioecológica (Carvalho-Barreto et al., 2009). Tais interações, no contexto familiar, conforme a duração, frequência, continuidade e intensidade com que ocorrem, possuem potencial para interferir diretamente nos processos proximais, afetando o desenvolvimento e podendo gerar competência ou disfunção nas pessoas envolvidas (Bronfenbrenner \& Evans, 2000).

O nível contextual de análise abarca as relações entre o sistema família e outros sistemas da comunidade (escola, vizinhança, unidades de saúde, etc; Carvalho-Barreto et al., 2009). Apesar de não terem se constituído como foco dessa investigação, algumas das informações coletadas acerca do bairro onde vivem as mulheres entrevistadas indicam que a violência urbana também se faz presente no cotidiano dessas famílias.

Ressalta-se que a investigação conduzida, através da amostragem probabilística e do baixo percentual de recusa, foi representativa de um contexto comunitário. Assim, retratou eventos que envolvem um delicado processo de detecção (Moura \& Reichencheim, 2005). Além disso, a pesquisa abordou diferentes relações de violência no sistema familiar ao invés identificar comportamentos isolados.

Dentre as limitações, a natureza das situações investigadas e o uso do domicílio como local de coleta dos dados podem ter contribuído para que algumas informações fossem subestimadas pelas respondentes. $\mathrm{O}$ fato de serem ouvidas apenas mulheres, a não investigação da frequência, motivação, severidade, co-ocorrência temporal dessas condutas, e do histórico familiar de violência, também são aspectos que restringem os achados dessa pesquisa.

\section{Considerações Finais}

Os dados obtidos indicam que a violência nas famílias é fenômeno prevalente e que pode manifestar-se em diferentes tipos de relação e direção num mesmo grupo familiar. Tal compreensão sugere a necessidade de que as práticas voltadas para a violência doméstica considerem não só o indivíduo, ou seja, agressor e/ou vítima identificados inicialmente, mas sua inserção dinâmica, num contexto de socialização e desenvolvimento. As relações estabelecidas com outros membros desse grupo, da comunidade, bem como com o cenário cultural no qual esses indivíduos encontram-se inseridos constituem importante aspecto na manifestação e abordagem do fenômeno da violência.

\section{Referências}

Abramsky, T., Watts, C. H., Garcia-Moreno, C., Devries, K., Kiss, L., Ellsberg, M., ...Heise, L. (2011, February). What factors are associated with recent intimate partner violence? Findings from the WHO multi-country study on women's health and domestic violence. BMC Public Health, 11 . Retrieved July 12, 2011, from http://www.biomedcentral. com/1471-2458/11/109

Archer, J. (2002). Sex differences in physically aggressive acts between heterosexual partners: A meta-analytic review. $A g$ gression and Violent Behavior, 7, 313-351.

Assis, S. G., Avanci, J. Q., Pesce, R. P., Pires, T. O., \& Gomes, D. L. (2012). Notificações de violência doméstica, sexual e outras violências contra crianças no Brasil. Ciência \& Saúde Coletiva, 17(9), 2305-2317.

Bandura, A. (2008). A evolução da teoria social cognitiva. In A. Bandura, R. G. Azzi, \& S. Polydoro (Eds.), Teoria social cognitiva: Conceitos básicos (pp. 15-41). Porto Alegre, RS: Artmed.

Bandura, A., Ross, D., \& Ross, S. A. (1961). Transmission of aggression through imitation of aggressive models. Journal of Abnormal and Social Psychology, 63, 575-582.

Bronfenbrenner, U. (1977). Toward an experimental ecology of human development. American Psychologist, 32(7), 513-531.

Bronfenbrenner, U. (1994). Ecological models of human development. In M. Gauvain \& M. Cole (Eds.), Readings on the development of de children (pp. 37-43). New York: Freeman.

Bronfenbrenner, U., \& Evans, G. W. (2000). Developmental science in the $21^{\text {st }}$ century: Emerging questions, theoretical models, research designs and empirical findings. Social Development, 9(1), 115-125.

Canha, J. (2008). A criança vítima de violência. In C. Machado \& R. A. Gonçalves (Eds.), Violência e vítimas de crimes. Crianças (pp. 17-37). Lisboa, Portugal: Quarteto.

Carvalho-Barreto, A., Bucher-Maluschke, J. S. N. F., Almeida, P. C., \& DeSouza, E. (2009). Desenvolvimento humano e violência de gênero: Uma integração bioecológica. Psicologia: Reflexão e Crítica, 22(1), 86-92.

Cecconello, A. M., De Antoni, C., \& Koller, S. H. (2003). Práticas educativas, estilos parentais e abuso físico no contexto familiar. Psicologia em Estudo (Maringá), 8, 45-54.

De Antoni, C., \& Koller, S. H. (2011). A pesquisa ecológica sobre violência no microssistema familiar. In S. H. Koller (Ed.), Ecologia do desenvolvimento humano: Pesquisa e intervenção no Brasil (pp. 315-339). São Paulo, SP: Casa do Psicólogo.

D’Oliveira, A. F. P. L., Schraiber, L. B., França, I., Jr., Ludermir, A. B., Portella, A. P., Diniz, S., ...Valença, O. (2009). Fatores associados à violência por parceiro íntimo em mulheres brasileiras. Revista de Saúde Pública, 43(2), 299-310.

Holt, S., Buckley, H., \& Whelan, S. (2008). The impact of exposure to domestic violence on children and young people: A review of the literature. Child Abuse \& Neglect, 32, 797-810. 
Instituto Brasileiro de Geografia e Estatística. (2000). Censo 2000. Recuperado em 16 de fevereiro, 2011, de http://www. ibge.gov.br/cidadesat/link.php? codmun $=313670$

Johnson, M. P. (2009). Differentiating among types of domestic violence: Implications for healthy marriages. In H. E. Peters \& C. M. K. Dusch (Eds.), Marriage and family: Perspectives and complexities (pp. 282-297). New York: Columbia University Press.

Krug, E. G., Dahlberg, L. L., Mercy, J. A., Zwi, A. B., \& Lozano, R. (Eds.). (2002). World report on violence and health. Geneva, Switzerland: World Health Organization.

Lamoglia, C. V. A., \& Minayo, M. C. S. (2009). Violência conjugal, um problema social e de saúde pública: Estudo em uma delegacia do interior do Estado do Rio de Janeiro. Ciência \& Saúde Coletiva, 14(2), 595-604.

Martins, C. B. G., \& Jorge, M. H. P. M. (2009). A violência contra crianças e adolescentes: Características epidemiológicas dos casos notificados aos Conselhos Tutelares e programas de atendimento em município do Sul do Brasil, 2002 e 2006. Epidemiologia e Serviços de Saúde (Brasília), 18(4), 315-334.

Minayo, M. C. S. (2005). Violência: Um problema para a saúde dos brasileiros. In Ministério da Saúde, Impacto da violência na saúde dos brasileiros (pp. 10-41). Brasília, DF: Ministério da Saúde.

Moraes, C. L., Hasselmann, M. H., \& Reichencheim, M. E. (2002). Adaptação transcultural do instrumento "Revised Conflict Tactic Scales (CTS2)" utilizado para identificar violência entre casais. Cadernos de Saúde Pública, 18(1), 163-176.

Moraes, C. L., \& Reichencheim, M. E. (2002). Cross-cultural measurement equivalence of the Revised Conflict Tactic Scales (CTS2): Portuguese version used to identify violence with couples. Cadernos de Saúde Pública, 18(3), 783-796.

Moura, A. T. M. S., \& Reichencheim, M. E. (2005). Estamos realmente detectando violência familiar contra a criança em serviços de saúde? A experiência de um serviço público do Rio de Janeiro, Brasil. Cadernos de Saúde Pública, 21(4), 1124-1133.

Oliveira, J. B., Lima, M. C. P., Simão, M. O., Cavariani, M. B., Tucci, A, M., \& Kerr-Corrêa, F. (2009). Violência entre parceiros íntimos e álcool: Prevalência e fatores associados. Revista Panamericana de Salud Pública, 26(6), 494-501.

Reichencheim, M. E., Dias, A. S., \& Moraes, C. L. (2006). Co-ocorrência de violência física conjugal e contra filhos em serviços de saúde. Revista de Saúde Pública, 40(4), 595-603.

Reichencheim, M. E., Klein, R., \& Moraes, C. L. (2007). Assessing the physical violence component of the Revised Conflict Tactic Scales when used in heterossexual couples: An item response theory analysis. Cadernos de Saúde Pública, 23(1), 53-62.

Reichencheim, M. E., \& Moraes, C. L. (2003). Adaptação transcultural do instrumento Parent-Child Conflict Tactic Scales (CTSPC) utilizado para identificar a violência contra a criança. Cadernos de Saúde Pública, 19(6), 1701-1712.

Reichencheim, M. E., \& Moraes, C. L. (2006). Psychometric properties of the Portuguese version of the Conflict Tactic Scales: Parent-Child version (CTSPC) used to identify child abuse. Cadernos de Saúde Pública, 22(3), 503-515.

Reichencheim, M. E., Moraes, C. L., Szklo, A., Hasselmann, M. H., Souza, E. R., Lozana, J. A., \& Figueiredo, V. (2006). The magnitude of intimate partner violence in Brazil: Portraits from 15 capital cities and the Federal District. Cadernos de Saúde Pública, 22(2), 425-437.
Salisbury, E. J., Henning, K., \& Holdford, R. (2009). Fathering by partner-abusive men: Attitudes on children's exposure to interparental conflict and risk factors for child abuse. Child Maltreatment, 14(3), 232-242.

Schraiber, L. B., D’Oliveira, A. F. P. L., França, I., Jr., Diniz, C. S., Portella, A. P., Ludermir, A. B., ...Couto, M. T. (2007). Prevalência da violência contra a mulher por parceiro íntimo em regiões do Brasil. Revista de Saúde Pública, 41(5), 797-807.

Schraiber, L. B., D’Oliveira, A. F. P. L., França, I., Jr., \& Pinho, A. A. (2002). Violência contra a mulher: Estudo em uma unidade de atenção primária à saúde. Revista de Saúde Pública, 36(4), 470-477.

Skinner, C. J., Holt, D., \& Smith, T. M. F. (1989). Analysis of complex surveys. Chichester, UK: John Wiley \& Sons.

Smith, P. H., Homish, G. G., Leonard, K. E., \& Cornelius, J. R. (2012). Intimate partner violence and specific substance use disorders: Findings from the national epidemiologic survey on alcohol and related conditions. Psychology of Addictive Behaviors, 26(2), 236-245.

Stelko-Pereira, A. C., \& Williams, L. C. A. (2010). Sobre o conceito de violência: Distinções necessárias. In L. C. A.Williams, J. M. D. Maia, \& K. S. A. Rios (Eds.), Aspectos psicológicos da violência: Pesquisa e intervenção cognitivo-comportamental (pp. 41-66). Santo André, SP: Esetec.

Straus, M. A. (2001). Scoring the CTS2 and CTSPC. Manchester, NH: University of New Hampshire. Retrieved February 14, 2011, from http://pubpages.unh.edu/ mas $2 /$ ctsb.htm

Straus, M. A., Hamby, S. L., Boney-McCoy, S., \& Sugarman, D. B. (1996). The Revised Conflict Tactic Scales (CTS2): Development and preliminary psychometric data. Journal of Family Issues, 17(3), 283-316.

Swan, S. C., Gambone, L. J., Caldwell, J. E., Sullivan, T. P., \& Snow, D. L. (2008). A review of research on women's use of violence with male intimate partners. Violence and Victims, 23(3), 301-314.

Testa, M., Livingston, J. A., \& Leonard, K. E., (2003). Women's substance use and experiences of intimate partner violence: A longitudinal investigation among a community sample. Addictive Behaviors, 28, 1649-1664.

Thompson, M. P., \& Kingree, J. B. (2006). The roles of victim and perpetrator alcohol use in intimate partner violence outcomes. Journal of Interpersonal Violence, 21(2), 163-177.

Vendruscolo, T. S., Ferriani, M. G. C., \& Silva, M. A. I. (2007). Public care policies for child and adolescent victims of domestic violence. Revista Latino-Americana de Enfermagem, $15,812-819$.

Vieira, L. J. E. S., Pordeus, A. M. J., Ferreira, R. C., Moreira, D. P., Maia, P. B., \& Saviolli, K. C. (2008). Fatores de risco para violência contra a mulher no contexto doméstico e coletivo. Saúde e Sociedade, 17(3), 113-125.

Zaleski, M., Pinsky, I., Laranjeira, R., Ramisetty-Mikler, S., \& Caetano, R. (2010). Violência entre parceiros íntimos e consumo de álcool. Revista de Saúde Pública, 44(1), 53-59.

Zanoti-Jeronymo, D. V., Zaleski, M., Pinsky, I., Caetano, R., Figli, N. B., \& Laranjeira, R. (2009). Prevalência de abuso físico na infância e exposição à violência parental em uma amostra brasileira. Cadernos de Saúde Pública, 25(11), 2467-2472. 